\title{
CONOCIMIENTO Y CRECIMIENTO COLOMBIANO: UNA APROXIMACIÓN A TRAVÉS DEL PROGRESO TECNOLÓGICO Y EL CAMBIO TÉCNICO*
}

\author{
GERMÁN SÁNCHEZ PÉREZ** \\ UNIVERSIDAD MILITAR NUEVA GRANADA
}

Recibido/ Received/ Recebido: 30-10-2011 - Aceptado/ Accepted / Aprovado: 27-11-2011

\begin{abstract}
Resumen
El artículo muestra que el conocimiento ha jugado un papel importante en la historia del crecimiento colombiano. Empleando las técnicas econométricas de co-integración, se muestra empíricamente, que el despegue y crecimiento de la economía colombiana desde 1905 hasta finales de la década de 1970, y su posterior disminución hasta finales del siglo XX está directamente relacionada con la aplicación o no del conocimiento tecnológico en la actividad productiva.
\end{abstract}

Palabras clave: Crecimiento económico, conocimiento, cambio técnico, progreso tecnológico.

\section{COLOMBIAN KNOWLEDGE AND GROWTH: AN APPROXIMATION THROUGH TECHNOLOGICAL PROGRESS AND TECHNICAL CHANGE}

\begin{abstract}
This article shows that knowledge had played an important role in Colombian growth history. Using co-integration econometrical techniques, this article shows empirically, that Colombian economy take-off and growing since 1905 until the end of 1970, and its posterior decreasing until the end of $\mathrm{XX}$ century is directly related to the application of technological knowledge in productive activity.

Keywords: Economic growth, knowledge, technical change, technological progress.
\end{abstract}

\footnotetext{
* Este documento hace parte del proyecto de investigación "conocimiento y crecimiento colombiano", financiado por la Universidad Militar "Nueva Granada", 2011. Además del autor, hacen parte del grupo de investigación el profesor de la Universidad Nacional de Colombia Leonardo Duarte, el matemático Edgar Malagón, los estudiantes Jennifer Rincón, Alejandro Blanco, Aydée Higuera, y la joven investigadora Angélica Buitrago. El autor agradece la participación y valiosa colaboración de cada uno de ellos, así como los comentarios de un evaluador anónimo; sin embargo, la responsabilidad por los errores y omisiones son exclusivas del autor.

** Candidato a doctor en economía de la Universidad Nacional de Colombia, Magíster en Economía de la Universidad de los Andes y Economista de la Universidad del Valle, docente investigador Universidad Militar "Nueva Granada". Correo electrónico: german. sanchez@unimilitar.edu.co,gsanchezpe1@yahoo.com.
} 


\title{
CONHECIMENTO E CRESCIMENTO COLOMBIANO: UMA APROXIMAÇÃO ATRAVÉS DO PROGRESSO TECNOLÓGICO E DA MUDANÇA TÉCNICA.
}

\begin{abstract}
Resumo
O artigo mostra que o conhecimento exerceu um papel importante na história do crescimento colombiano. Empregando as técnicas econométricas de cointegração, mostra-se empiricamente que a decolagem e o crescimento da economia colombiana de 1905 até finais da década de 70, e sua posterior diminuição até finais do século XX, está diretamente relacionada com a aplicação ou não do conhecimento tecnológico na atividade produtiva.
\end{abstract}

Palavras chave: Crescimento econômico, Conhecimento, Mudança técnica, Progresso tecnológico.

Sanchéz, G. (2011) Conocimiento y crecimiento colombiano: una aproximación a través del progreso tecnológico y el cambio técnico. En: Revista de la Facultad de Ciencias Económicas de la Universidad Militar Nueva Granada. rev.fac.cienc.econ, XIX (2).

JEL: O30, O31, O33.

\section{Introducción}

El desarrollo alcanzado por algunas economías del mundo está asociado con tasas positivas y sostenidas de crecimiento en el largo plazo, importantes mejoras en la distribución del producto, y el aumento del nivel de capacidades y oportunidades de la población (Barro \& Sala-i-Martín, 1995; Sen, 1987, 1996, 2000; Ray, 1988). El estudio de las causas del crecimiento económico sostenido se ha convertido, entonces, en un tema central de investigación de la ciencia económica. Y entre los determinantes fundamentales del crecimiento económico moderno, el conocimiento es considerado hoy como un elemento esencial. Así, el estudio de los efectos del conocimiento sobre el crecimiento económico ha venido ganando importancia en los últimos años, tanto desde la teoría como desde la empírea.

Desde el punto de vista teórico, desde el siglo XVIII, con los economistas clásicos, la economía ha resaltado la importancia que tiene el avance del conocimiento tecnológico en el desarrollo económico. Smith (1992) por ejemplo, destacó la relación que existe entre el avance del conocimiento tecnológico, el crecimiento y el bienestar (Benavides, 2010,
66), y Marx (1984) destacó el papel clave que juega el conocimiento tecnológico en el avance y contradicciones del desarrollo del sistema capitalista. El análisis económico más reciente del crecimiento económico de largo plazo, parte de las aportaciones seminales de Marx y Schumpeter y lo vincula con la innovación tecnológica (Torrent, 2009; Benavides, 2010; Salazar, 2010). Trabajos pioneros como los de Schumpeter (1934) sobre el empresario innovador, de Schultz (1961) sobre el capital humano, Arrow (1962) sobre el aprender haciendo y trabajos posteriores como los de Romer (1986; 1990), Lucas (1998), Aghion \& Howitt (1998; 2006), Grossman \& Helpman (1991), CEPAL (1999) y OECD (1996) muestran que el conocimiento afecta de manera positiva al crecimiento económico.

Desde el punto de vista empírico, trabajos como los de Vilaseca \& Torrent (2005), Barceló (2001), CEPAL (1999; 2008a; 2008b), CEPAL \& Naciones Unidas (2009) y Banco Mundial (2003), muestran que desde las dos últimas décadas del siglo $\mathrm{XX}$, éste es uno de los factores que más ha influido sobre el crecimiento de las economías desarrolladas.

Una revisión de la literatura económica que se ocupa de la relación conocimiento y crecimiento en el 
mundo, en general, y sobre Colombia, en particular, muestra una serie de indicadores sobre conocimiento que entidades como la CEPAL, el Banco Mundial, el DNP y el Observatorio Colombiano de Ciencia y Tecnología (OCyT) han venido construyendo recientemente ${ }^{1}$. Estos indicadores muestran que en Colombia, los efectos del conocimiento sobre el crecimiento no han sido tan importantes como en los países desarrollados, y en América Latina, Colombia es uno de los países donde, tanto el Estado como las empresas, menos se ha aprovechado este factor.

Una mirada al crecimiento económico colombiano, muestra que el siglo XX es el de mayor progreso en toda su historia económica. Un crecimiento del PIB de $4,7 \%$, a precios de 1975 , frente a un crecimiento de la población de $2,34 \%$, significaron un mejoramiento en el bienestar de la población colombiana durante este siglo, que, sin embargo, no fue suficiente para alcanzar niveles de bienestar similares a los de las economías desarrolladas (GRECO, 2002).

La historia del crecimiento de la economía colombiana, en general, y de la industria, en particular, presenta dos sub-periodos claramente diferenciados durante el siglo $\mathrm{XX}^{2}$. Un periodo de un crecimiento promedio de $5,04 \%$, progreso tecnológico $(2,17 \%)$, y avance industrial, que va desde 1905 hasta finales de la década de 1970. Otro periodo de disminución del crecimiento $(3,56 \%)$ y del progreso tecnológico (1,58\%), y truncamiento del progreso industrial, que va desde finales de la década de 1970 hasta entrado el siglo XXI (ver Ilustración 1).

En este contexto del crecimiento económico colombiano y mundial, donde se reconoce al conocimiento como el principal factor que impulsa el crecimiento moderno $^{3}$, surgen algunas inquietudes como, por ejemplo, ¿qué tanto el crecimiento colombiano, su tendencia decreciente y variaciones observadas, se relacionan o son explicadas por la aplicación o no del conocimiento en la actividad productiva? De este interrogante nos ocupamos en este documento.

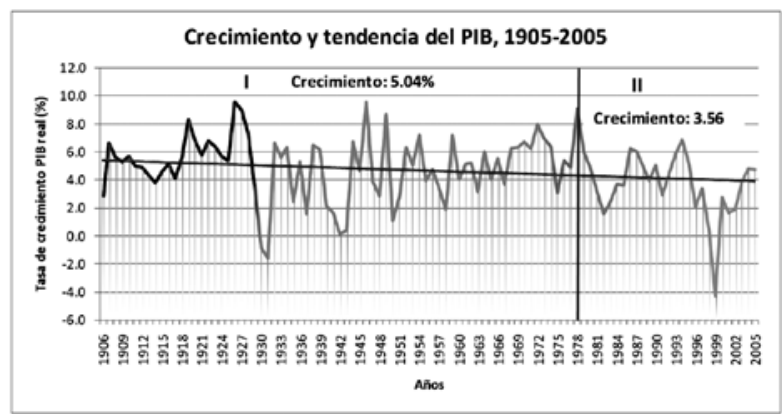

Ilustración 1. Crecimiento del PIB real y tendencia lineal, 1905-2005 (Precios de 1975). ${ }^{4}$

\section{Conocimiento y crecimiento colombiano}

En Colombia existen algunos indicadores recientes que se ocupan de la relación conocimiento y crecimiento económico, que nos permiten hacer una aproximación al tema. Sin embargo, no existe información estadística (series) que permita estimar de manera directa los efectos del conocimiento sobre el crecimiento histórico colombiano. Una solución alternativa a este inconveniente se encuentra en el concepto mismo de conocimiento desde el punto de vista económico, y el marco de análisis neoclásico de crecimiento.

Como lo expresa Morín (1998, 20), "El conocimiento es sin duda un fenómeno multidimensional en el sentido que, de manera inseparable, a la vez es

1 La primera encuesta formal sobre desarrollo e innovación tecnológica en Colombia (EDIT 1) se realizó en 1996.

2 En este mismo sentido, autores como Ortiz et. Al. (2009), por ejemplo, encuentran que la senda de desarrollo en Colombia en los últimos ochenta años exhibe dos grandes etapas: de 1932 a 1979 el país experimenta un proceso de industrialización, posteriormente, y hasta la actualidad, el país sufre un proceso de desindustrialización; y Ocampo (1999) muestra que, después de la segunda guerra mundial, se pueden identificar dos grandes etapas en la industria colombiana: la primera desde 1945 hasta 1974, donde la industria fue el centro de atención de la política económica y vivió su mejor época, y la segunda etapa desde 1974 hasta 1983, donde la industria experimentó una crisis creciente.

3 OECD, (1996); Vilaseca \& Torrent (2005), Torrent (2009), Barceló (2001), CEPAL (2008, 2009), CEPAL \& Naciones Unidas (2009), Banco Mundial (2003), entre otros.

4 Fuente: Elaborado con base en datos de Ortiz et al (2009) y GRECO (2002). 
físico, biológico, cerebral, mental, psicológico, cultural, social". Sin embargo, desde el punto de vista económico se reconoce que el progreso tecnológico, el cambio técnico y la innovación en general, son expresiones de la aplicación o de la materialización del conocimiento en la actividad productiva.

De otro lado, el marco de análisis neoclásico permite relacionar teóricamente en el largo plazo el crecimiento económico con el capital, el trabajo, y el cambio técnico ${ }^{5}$, siendo esta última una variable exógena que recoge el efecto sobre el crecimiento del conocimiento y demás factores diferentes al capital y al trabajo. Además, a partir del modelo teórico, podemos formular un modelo econométrico con el que se puede estimar la relación en el largo plazo entre el cambio técnico y el crecimiento; se puede estimar, igualmente, la tasa de progreso tecnológico y su efecto sobre el producto por trabajador o productividad del trabajo.

Finalmente, en el país existe información estadística que permiten realizar las estimaciones econométricas que relacionan el crecimiento con el cambio técnico desde 1925. Así, aunque el marco de análisis neoclásico no es el más adecuado para analizar las causas históricas del comportamiento económico y su relación con el conocimiento, si nos permite hacer estimaciones empíricas que se constituye en información valiosa que nos ayuda a entender de manera indirecta los efectos del conocimiento sobre el crecimiento.

En seguida se formula lo anunciado, bajo el marco de análisis tradicional de crecimiento exógeno que parte de Solow $(1956,1957)$ y Swan (1956). En la parte uno, se examinan los efectos del progreso tecnológico sobre el producto por trabajador o crecimiento de la productividad del trabajo. En la parte dos, se mira el efecto del cambio técnico en el crecimiento del producto total. Y en la parte tres, se presenta las conclusiones. Las estimaciones se realizan empleando las técnicas de co-integración, lo que permite estimar parámetros in-sesgados, y la información estadística es tomada de Ortiz et al. (2009), GRECO (2002) y el DANE.

\subsection{Progreso tecnológico y producto por trabajador}

\subsubsection{Consideraciones teóricas}

El estudio de la relación cambio técnico, progreso tecnológico y crecimiento no es nuevo en la literatura económica. $Y$ hoy se reconoce que existe una relación directa entre avances de los conocimientos científicos y tecnológicos, innovación, productividad del trabajo y crecimiento (Amable, 2003).

La formalización teórica - matemática y análisis empírico de la relación cambio técnico, progreso tecnológico y crecimiento económico parte con los trabajos de mediados del siglo XX de Solow-Swan. Bajo este enfoque ${ }^{6}$ se puede relacionar al crecimiento con el capital y el progreso tecnológico. Y es posible, a partir del modelo teórico, formular y estimar un modelo econométrico de co-integración que nos permita determinar empíricamente el efecto del progreso tecnológico en la producción por trabajador o productividad laboral. El empleo de la técnica de co-integración como método de estimación, tiene importantes implicaciones en la obtención de los resultados. Con este método se puede estimar de manera directa la tasa del progreso tecnológico, con la ventaja que los coeficientes o parámetros que se obtienen no presentan los problemas de sesgo de las técnicas de estimación tradicionales.

\subsubsection{El modelo}

Bajo el marco teórico neoclásico Solow-Swan de crecimiento exógeno, podemos suponer que la producción colombiana se ajusta a una función de producción del tipo Cobb-Douglas. En tiempo discreto, y suponiendo que el producto $(\mathrm{Y})$ depende del capital (K) del trabajo (L) y de un factor tecnológico que au-

\footnotetext{
En la literatura, el cambio técnico es conocido también como el "residuo de Solow", productividad total de los factores o PTF.

6 Hay que resaltar, sin embargo, que esta aproximación, como cualquier otra, presenta deficiencias (Aghion \& Howitt (1998 y 2006), entre las que se resaltan que la tecnología es considerada como homogénea y no se explica cómo se genera y cómo la adoptan las empresas, y el contexto no influye en la evolución y dinámica del cambio técnico y el progreso tecnológico.
} 
menta la eficiencia laboral (A), la función de producción sería (Obstfeld \& Rogoff, 1996; GRECO, 2002):

$Y t+1=K t+1 \propto A t+1(11-\propto) L t+11-\propto$

Con Y: PIB; K: capital; L: PEA; A: factor o parámetro tecnológico que aumenta la eficiencia laboral, $\alpha$ : participación de $K$ en $Y, 0<\alpha<1 ; t+1$ : un periodo cualquiera, rendimientos marginales decrecientes del capital y rendimientos constantes de escala.

Si suponemos que el parámetro tecnológico que aumenta la eficiencia laboral (A) sigue una evolución en el tiempo como la siguiente:

$A t+1=A t(1+g a) 1-\propto$

Donde: ga es la tasa de crecimiento por periodo del parámetro $A$ o tasa de crecimiento del progreso tecnológico.

Reemplazando [2] en [1], tenemos:

$Y t+1=K t+1 \propto A t 1+a 1-\propto L t+11-\propto$

En tiempo continuo (3) quedaría:

$Y=K \alpha A O(1+a)(1-\alpha) L(1-\alpha)$

En términos per cápita (dividiendo por L),

$y t=A O(1+g a)(1-\alpha) t k t \alpha$

Donde: $y t=Y t L t ; k t=K t L t$.

$y t=$ PIB real dividido por la población económicamente activa (PEA);

$A 0$ = factor o parámetro tecnológico que aumenta la eficiencia de la población laboral en el momento inicial $(\mathrm{t}=0)$;

$g a=$ tasa de crecimiento del parámetro A o progreso tecnológico;

$k t=$ capital real dividido por la PEA;

$\alpha=$ elasticidad del producto al capital.

\subsubsection{Especificación del VEC.}

Nuestro propósito es estimar la tasa del progreso tecnológico (ga). En el caso de series no estacionarias, que son integradas de orden $1(I(1))$ y que están co-integradas, como es el caso del producto por trabajador y el capital por trabajador en Colombia (ver anexo 1), la forma más adecuada para estimar ga es un modelo de co-integración o, de manera más específica, un modelo de corrección de errores (VEC, por sus siglas en inglés). En consecuencia, en seguida formulamos y estimamos un VEC entre el logaritmo natural de producto por trabajador y el logaritmo natural del capital por trabajador. Para esto expresamos la ecuación [5] en logaritmos:

$y t=\gamma+1-\alpha \delta t+\alpha k t$

Siendo $y t=\ln y t ; \gamma=\ln A 0 ; \delta=\ln 1+g a ; k t=\ln k t$

Si el análisis de las series muestra que existe un vector de co-integración, podemos formular y estimar un VEC, que incluye relaciones tanto de largo como de corto plazo. A partir de la ecuación [6] y el análisis de las series (ver anexo 1), podemos formular el VEC de la siguiente forma, con intercepto, tendencia y un rezago:

$\Delta y t=\theta 11 \Delta y t-1+\theta 12 \Delta k t-1+\pi 1 y t-1-\alpha k t-1-\gamma-\beta t+\varepsilon 1 t[7]$ $\Delta k t=\theta 21 \Delta y t-1+\theta 22 \Delta k t-1+\pi 2 y t-1-\alpha k t-1-\gamma-\beta t+\varepsilon 2 t$

Con $\pi 1 ; \pi 2 \leq 0$. Donde:

$\beta=1-\alpha \delta$

Ahora, despejando $\delta$, obtenemos:

$\delta=\beta 1-\alpha$

Dado que $\delta=\ln 1+$ ga, solucionando la ecuación y despejando obtenemos ga: ga $=e \delta-1$

Los coeficientes $\theta 11, \theta 12, \theta 21, \theta 22$ reflejan el impacto de corto plazo de cambios en las variaciones en $t-1 ; \pi 1, \pi 2$ representan las velocidades de ajuste a un desequilibrio entre el producto por trabajador $y$ sus determinantes de largo plazo; $\alpha, \gamma$ y $\beta$ son los coeficientes de largo plazo.

Nótese que tenemos un marco de análisis donde el crecimiento de la productividad del trabajo está rela- 
cionado con el crecimiento del capital por trabajador y la tasa de crecimiento del factor de productividad. Además, una vez corrido el VEC, podemos estimar tasa de crecimiento por periodo de A o progreso tecnológico ecuación [10].

\subsubsection{Consideraciones empíricas}

\section{Estimación modelos VEC}

Para estimar el modelo, tomamos los datos de Ortiz et al. (2009), GRECO (2002) y DANE 7 . En el anexo 1 se muestra que las series del producto por trabajador y capital por trabajador no son estacionarias, son integradas de orden 1 (I(1)) y están co-integradas. Empleando el método de Johansen (1988; 1995), y una vez realizadas las pruebas formales correspondientes, se encontró que el VEC que se debe estimar para todo el periodo de análisis, 1924-2005, debe contener constante, tendencia, dos variables dummy y un rezago; el VEC para el sub-periodo 1924-1979 debe contener constante, tendencia y un rezago; y el VEC para el sub-periodo 1969-2005 debe contener constante, tendencia, una dummy y un rezago.

Nótese que los dos sub-periodos se traslapan entre 1969 y 1979. Esto se debe a que en el último subperiodo, las series resultaron estar co-integradas sólo entre 2005-1969 y hacia atrás (es decir, un periodo mínimo de 37 años ${ }^{8}$ ). Desde el punto de vista de la evolución del conjunto de políticas y hechos históricos que se relacionan con el crecimiento de la economía colombiana esta situación es congruente. En Colombia, desde 1925 y hasta mediados de la década de 1970 se tomaron un conjunto de medidas como la protección a la industria naciente, el estímulo a la importación de maquinaria y equipo, el modelo de sustitución de importaciones, entre otras, que impulsaron la introducción de nuevas tecnologías y ampliaron el mercado interno, lo que coadyuvó a que se fuese configurando un sistema social de innovación y producción (SSIP) que favoreció el desapegue y crecimiento del sector industrial y de la economía en general. Desde la década de 1960, con la política de promoción de exportaciones, en el país se empezó a tomar un conjunto de medidas que marcaron el inicio del desmonte paulatino de la protección a la industria naciente, se restringió el mercado interno y se adoptó un nuevo modelo de desarrollo basado en el mercado, lo que significó un cambio en el estado del sistema social de innovación y producción (o cambio en el marco institucional) que marcó un quiebre del dinamismo que traía el sector industrial y ha significado una disminución del crecimiento. Como se puede percibir, no se pasó de un momento a otro de un estado del sistema social de innovación y producción a otro nuevo, lo que llevó a que los efectos sobre el crecimiento de los diferentes marcos institucionales (SSIP) en este periodo se entrelacen y que los periodos de análisis se traslapen.

Los resultados del VEC para todo el periodo de análisis y los dos sub-periodos, donde las series están co-integradas, se presentan en seguida.

En la tabla 1 se presenta los resultados de la estimación del VEC para todo el periodo de análisis (19242005). El análisis de los residuos del VEC permite concluir que son Gauss-Markov; es decir, no están autocorrelacionados, son normales y el sistema es marginalmente estable (en el VAR el sistema es estable).

En la tabla 2 se presenta los resultados del VEC para el sub-periodo 1924-1975. El análisis de los residuos del VEC permiten concluir que son Gauss-Markov; es decir, no están autocorrelacionados, son normales y el sistema es marginalmente estables (En el VAR el sistema es estable).

En la tabla 3 se presenta los resultados del VEC para el sub-periodo 1969-2005. El análisis de los residuos del VEC permiten concluir que son Gauss-Markov; es decir, no están autocorrelacionados, son normales y el sistema es marginalmente estables (En el VAR el sistema es estable).

www.dane.gor.co

Si tenemos en cuenta que el análisis de co-integración es para periodos largos esta situación era de esperarse. 
Tabla 1. VEC periodo 1924 - 2005: modelo con constante, tendencia, dos dummy y un rezago 9 .

\begin{tabular}{|c|c|c|}
\hline \multicolumn{2}{|c|}{ Vector Error CorrectionEstimates } & \\
\hline \multicolumn{2}{|c|}{ Date: 03/31/11 Time: 07:01 } & \\
\hline \multicolumn{2}{|c|}{ Sample (adjusted): 19272005} & \\
\hline \multicolumn{2}{|c|}{$\begin{array}{l}\text { Includedobservations: } \\
79 \text { afteradjustments }\end{array}$} & \\
\hline \multicolumn{3}{|c|}{ Standard errors in ( ) \& t-statistics in [ ] } \\
\hline CointegratingEq: & CointEq1 & \\
\hline LNYPEA $(-1)$ & 1.000000 & \\
\hline \multirow[t]{3}{*}{$\operatorname{LNKPEA}(-1)$} & -0.655144 & \\
\hline & $(0.05615)$ & \\
\hline & {$[-11.6686]$} & \\
\hline \multirow[t]{3}{*}{ @TREND(24) } & -0.006200 & \\
\hline & $(0.00112)$ & \\
\hline & {$[-5.52729]$} & \\
\hline C & 0.114613 & \\
\hline Error Correction: & D(LNYPEA) & D(LNKPEA) \\
\hline \multirow[t]{3}{*}{ CointEq1 } & -0.009183 & 0.713722 \\
\hline & $(0.03146)$ & $(0.12836)$ \\
\hline & {$[-0.29188]$} & [ 5.56043] \\
\hline \multirow[t]{3}{*}{$\mathrm{D}(\operatorname{LNYPEA}(-1))$} & 0.116797 & 1.232173 \\
\hline & $(0.11092)$ & $(0.45251)$ \\
\hline & {$[1.05300]$} & [ 2.72298] \\
\hline \multirow[t]{3}{*}{ D(LNKPEA $(-1))$} & 0.042833 & 0.259567 \\
\hline & $(0.02480)$ & $(0.10116)$ \\
\hline & [1.72733] & [ 2.56583] \\
\hline \multirow[t]{3}{*}{ C } & 0.016438 & -0.005540 \\
\hline & $(0.00311)$ & $(0.01270)$ \\
\hline & [ 5.28132] & {$[-0.43631]$} \\
\hline \multirow[t]{3}{*}{ D98 } & -0.163367 & -0.211605 \\
\hline & $(0.02119)$ & $(0.08645)$ \\
\hline & {$[-7.70941]$} & {$[-2.44771]$} \\
\hline \multirow[t]{3}{*}{ D99 } & -0.057898 & -0.191970 \\
\hline & $(0.02734)$ & $(0.11152)$ \\
\hline & {$[-2.11796]$} & {$[-1.72135]$} \\
\hline R-squared & 0.543707 & 0.505584 \\
\hline
\end{tabular}

Tabla 2. VEC periodo 1924-1979: modelo con constante, tendencia, y un rezago ${ }^{10}$.

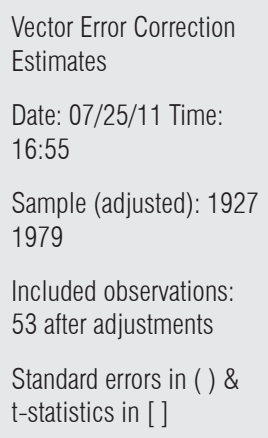

\section{Cointegrating Eq:}

CointEq1

\begin{tabular}{lc}
\hline LNYPEA(-1) & 1.000000 \\
LNKPEA(-1) & -0.453334 \\
& $(0.06928)$ \\
& {$[-6.54383]$} \\
@TREND(24) & -0.011746 \\
& $(0.00174)$ \\
& {$[-6.74161]$} \\
C & 1.374847
\end{tabular}

Error Correction: D(LNYPEA)
D(LNKPEA)

\begin{tabular}{lcc}
\hline CointEq1 & -0.035950 & 1.215187 \\
& $(0.06935)$ & $(0.29525)$ \\
& {$[-0.51842]$} & {$[4.11581]$} \\
D(LNYPEA(-1)) & 0.068738 & 1.169618 \\
& $(0.13751)$ & $(0.58546)$ \\
& {$[0.49989]$} & {$[1.99779]$} \\
D(LNKPEA(-1)) & 0.048840 & 0.260587 \\
& $(0.03302)$ & $(0.14059)$ \\
& {$[1.47912]$} & {$[1.85358]$} \\
C & 0.020311 & -0.014207 \\
& $(0.00442)$ & $(0.01882)$ \\
& {$[4.59500]$} & {$[-0.75489]$} \\
\hline R-squared & 0.098106 & 0.305682 \\
Adj. R-squared & 0.042888 & 0.263173
\end{tabular}


Tabla 3. VEC periodo 1969 - 2005: modelo con constante, tendencia, una dummy y un rezago ${ }^{11}$.

\begin{tabular}{|c|c|c|}
\hline $\begin{array}{l}\text { Vector Error Correction } \\
\text { Estimates }\end{array}$ & & \\
\hline $\begin{array}{l}\text { Date: 06/30/11 Time: } \\
\text { 14:18 }\end{array}$ & & \\
\hline Sample: 19692005 & & \\
\hline $\begin{array}{l}\text { Included observations: } \\
37\end{array}$ & & \\
\hline $\begin{array}{l}\text { Standard errors in ( ) \& } \\
\text { t-statistics in [ ] }\end{array}$ & & \\
\hline Cointegrating Eq: & CointEq1 & \\
\hline $\operatorname{LNYPEA}(-1)$ & 1.000000 & \\
\hline LNKPEA $(-1)$ & -0.613868 & \\
\hline & $(0.07518)$ & \\
\hline & {$[-8.16541]$} & \\
\hline @TREND(24) & -0.006048 & \\
\hline & $(0.00139)$ & \\
\hline & {$[-4.35872]$} & \\
\hline C & 0.285105 & \\
\hline Error Correction: & D(LNYPEA) & D(LNKPEA) \\
\hline CointEq1 & -0.001112 & 0.705650 \\
\hline & $(0.04272)$ & $(0.17461)$ \\
\hline & {$[-0.02602]$} & [ 4.04133] \\
\hline D(LNYPEA $(-1))$ & 0.453728 & 1.727471 \\
\hline & $(0.11357)$ & $(0.46417)$ \\
\hline & [ 3.99517$]$ & [3.72160] \\
\hline $\mathrm{D}(\mathrm{LNKPEA}(-1))$ & -0.017837 & 0.277155 \\
\hline & $(0.03699)$ & $(0.15118)$ \\
\hline & {$[-0.48222]$} & [1.83324] \\
\hline C & 0.009442 & -0.005580 \\
\hline & $(0.00299)$ & $(0.01221)$ \\
\hline & [ 3.16154$]$ & {$[-0.45716]$} \\
\hline D98 & -0.163066 & -0.212495 \\
\hline & $(0.01743)$ & $(0.07123)$ \\
\hline & {$[-9.35715]$} & {$[-2.98337]$} \\
\hline R-squared & 0.777230 & 0.659010 \\
\hline Adj. R-squared & 0.749383 & 0.616386 \\
\hline
\end{tabular}

Con estos resultados se puede determinar empíricamente el papel que ha jugado el progreso tecnológico en el crecimiento del producto por trabajador o productividad del trabajo; en particular, qué tanto explica y se relaciona con el crecimiento de la productividad en el largo plazo, el mayor crecimiento observado hasta finales de la década de 1970, y la desaceleración observada a partir de esta misma década.

\section{i.i. Cálculo y análisis de los resultados}

En la tabla 4 se presenta el crecimiento del producto total y por trabajador (productividad laboral), del capital total y por trabajador, y del trabajo ${ }^{12}$ para el periodo 1924-2005 y los sub-periodos 1924-1979 y 1969-2005. Estos datos muestran: primero, un crecimiento positivo del producto y del producto por trabajador colombiano desde 1924 hasta el 2005, que en promedio fue de $4,5 \%$ y de $1,76 \%$ respectivamente. Segundo, el crecimiento tanto del Producto total como del producto por trabajador no han sido constantes $y$ sufren una desaceleración desde finales de la década de 1970, aunque la desaceleración fue más dramática en el producto por trabajador: el crecimiento promedio del producto total entre 1925 y 1979 fue 4,8\%, mientras el crecimiento entre 1969 y 2005 fue de 4,4\%; el crecimiento del producto por trabajador, en los mismos periodos, fue de $2,46 \%$ y $0,98 \%$ respectivamente. Tercero, el crecimiento del producto total, el capital, y el producto por trabajador fueron mayores a principios que a final del siglo, mientras que el crecimiento de la PEA fue mayor a final que a comienzos de siglo. Cuarto, el crecimiento del capital por trabajador ha sido positivo pero ha venido disminuyendo: pasó de 2,6\% entre 1924-1979 a un 1,2\% entre 1969-2005.

En la tabla 5 se muestra la tasa del progreso tecnológico (ga), la participación del capital en el producto $(\alpha)$, y el crecimiento del producto por trabajador para el periodo 1924 - 2005 y los sub-periodos 1294-1979 y 1969-2005. Los cálculos de ga para el periodo completo y los sub-periodos se realizaron con base en la ecuación [9] y [10] y en los resultados de los VEC estimados con la técnica de co-integración de Johansen (tablas 1, 2, y 3).

1 Datos de la investigación.

12 Población ecónomamente activa, PEA. 
Tabla 4. Crecimiento del producto total y por trabajador, capital total y por trabajador, y trabajo, 1905 - 2005. (Variaciones porcentuales medias) $)^{13}$

\begin{tabular}{|c|c|c|c|c|c|}
\hline Periodos & $\boldsymbol{\Delta}$ PIB & $\boldsymbol{\Delta K}$ & $\boldsymbol{\Delta}$ PEA & $\boldsymbol{\Delta}$ (PIB/PEA) & $\boldsymbol{\Delta}(\mathbf{K} /$ PEA) \\
\hline $1924-1979$ & 4,81 & 4,97 & 2,35 & 2.46 & 2.61 \\
\hline $1969-2005$ & 4,37 & 4,57 & 3,41 & 0.98 & 1.20 \\
\hline $1924-2005$ & 4,48 & 4,77 & 2,70 & 1.76 & 2.05 \\
\hline
\end{tabular}

Tabla 5. Progreso tecnológico, ga, participación del capital en el producto, $\alpha$, y crecimiento del producto por trabajador, 1924-2005..$^{14}$

\begin{tabular}{|c|c|c|c|c|c|}
\hline Periodo & B & $\boldsymbol{\delta}=\frac{\beta_{t}}{(1-\alpha)}$ & A & $g_{a}=\left(e^{\hat{\delta}}-1\right)$ & $\boldsymbol{\Delta}(\mathbf{Y} /$ PEA $)$ \\
\hline $1924-1979$ & 0,011568 & 0,021187 & 0,453 & 2,17 & 2.46 \\
\hline $1969-2005$ & 0.006048 & 0.015663 & 0.614 & 1.58 & 0.98 \\
\hline $1924-2005$ & 0,006200 & 0,017971 & 0,655 & 1,81 & 1.76 \\
\hline
\end{tabular}

Los resultados de la tabla 5 muestra que en el largo plazo (1924-2005), la tasa del progreso tecnológico fue levemente superior al crecimiento del producto por trabajador, de $1,81 \%$ y de $1,76 \%$ respectivamente. Sin embargo, esta situación no se presenta entre los dos sub-periodos analizados: mientras a comienzos del siglo XX y hasta finales de la década de 1970, el crecimiento del producto por trabajador fue superior a la tasa de crecimiento de la productividad del trabajo, de $2,46 \%$ y $2,17 \%$ respectivamente; a finales de este mismo siglo y comienzos del XXI, el crecimiento del producto por trabajador fue inferior a la tasa de crecimiento de la productividad del trabajo, $0,98 \%$ y $1,58 \%$ respectivamente.

Estos datos arrojan luz sobre dos importantes aspectos del crecimiento del producto por trabajador o productividad del trabajo en Colombia: (i) sugieren que en Colombia se cumple la hipótesis de que en el largo plazo, debido a los rendimientos marginales decrecientes, los efectos del capital sobre el producto por trabajador se agotan, y el crecimiento de éste último depende de la tasa del progreso tecnológico. En otras palabras, es la dinámica del progreso tecnológico la que explica la productividad del trabajo en el largo plazo en Colombia. (ii) un crecimiento del progreso tecnológico superior al crecimiento del producto por trabajador en las últimas décadas del siglo XX, indica que la disminución del capital por trabajador (ilustración 2) también afectó de manera negativa el crecimiento del producto por trabajador en este periodo.

Los datos de la tabla 5 muestran, igualmente, que la tasa de crecimiento del progreso tecnológico no ha permanecido constante y ha venido disminuyendo en el tiempo: de un crecimiento de $2.17 \%$ entre $1924-$ 1979 se pasó a un crecimiento de $1.58 \%$ entre 1969 2005. Si como acabamos de mostrar, la dinámica de la productividad del trabajo depende en el largo plazo de la dinámica del progreso tecnológico, esta situación es preocupante y explica, en gran parte, la dismi-

13 Fuente: El crecimiento del PIB es con base en Ortiz et al. (2009); de K con base a GRECO (2002) y a partir de 1998 la serie se empató con las tasas de crecimiento del DANE. PEA: 1905-1997 de GRECO (2002), de 1998-2000 tomada del DANE; de 2002-2005, tomada del OCCyT (2007)

14 Fuente: cálculos propios con base en los modelos de co-integración. La fuente de PIB y K son Ortiz et al (2009) y DANE; la de PEA se extrajo de GRECO (2002) y DANE. 
nución del crecimiento colombiano desde finales de la década de 1970 hasta finales del siglo XX.

El capital por trabajador no explica la productividad del trabajo en Colombia en el largo plazo, pero si tiene efectos en el corto y mediano plazo, y sus efectos fueron más importantes a finales que a comienzos de siglo. Obsérvese en la tabla 5 que la elasticidad del capital respecto al producto ha venido aumentando en el tiempo: fue de $0.45 \%$ para el primer subperiodo y de $0.61 \%$ para el segundo sub-periodo ${ }^{15}$. Esta situación está relacionada con la disminución de la dinámica del progreso tecnológico y es claramente contraria a lo que ha venido pasando en las economías que presentan en el mundo las mayores tasas de crecimiento desde las dos últimas décadas del siglo XX, donde es el conocimiento, la ciencia y la tecnología, y la innovación los factores más importantes para el crecimiento (Vilaseca \& Torrent, 2005; OECD, 1996).

Para examinar de manera más precisa el papel de la tasa de crecimiento del progreso tecnológico en el crecimiento del producto por trabajador, debemos tener en cuenta que, en el marco empleado, el crecimiento puede deberse a (Blanchard, 2008): (i) Un aumento de la tasa de crecimiento del progreso tecnológico, debida a la aplicación de los avances de los conocimientos científicos y tecnológicos en la actividad productiva. En este caso, el crecimiento re- fleja un crecimiento equilibrado; es decir, en el largo plazo, si el progreso tecnológico es mayor (menor), también lo será el crecimiento (decrecimiento) equilibrado de la producción. En consecuencia, las tasas de crecimiento del producto por trabajador y la tasa del progreso tecnológico deben ser iguales o similares. En el caso colombiano, donde se presenta un decrecimiento del producto por trabajador a finales de siglo, debe presentarse un decrecimiento igual o similar del progreso tecnológico. (ii) Un nivel más alto del capital efectivo por trabajador. Este ajuste lleva a un periodo de mayor crecimiento del producto por trabajador, aunque la tasa de progreso tecnológico no haya aumentado o incluso haya disminuido. En este caso, la tasa de crecimiento del producto por trabajador debe ser superior a la tasa de progreso tecnológico. Ahora, cuando hay un decrecimiento del producto por trabajador, ésta debe ser mayor a la disminución de la tasa del progreso tecnológico; es decir, la caída de la productividad es explicada básicamente por la caída de la inversión del capital por trabajador.

En la tabla 6, se presenta la tasa de crecimiento del producto por trabajador y la tasa de progreso tecnológico y sus variaciones para los dos sub-periodos estudiados. De alli se puede extraer lo siguiente: Primero, el periodo de mayor crecimiento del producto por trabajador observado en la economía colombiana, conocido como de industrialización,

Tabla 6. Crecimiento del producto por trabajador y tasa de progreso tecnológico' ${ }^{16}$

Crecimiento del producto por trabajador

\begin{tabular}{|c|c|c|c|c|c|}
\hline $\begin{array}{c}1924-1979 \\
(1)\end{array}$ & $\begin{array}{c}1969-2005 \\
(2)\end{array}$ & $\begin{array}{c}\text { Variación } \\
(3=2-1)\end{array}$ & $\begin{array}{c}1924-1979 \\
(4)\end{array}$ & $\begin{array}{c}1969-2005 \\
(5)\end{array}$ & $\begin{array}{c}\text { Variación } \\
(6=5-4)\end{array}$ \\
\hline 2,46 & 0.98 & -1.48 & 2,17 & 1,58 & -0.59 \\
\hline
\end{tabular}

15 Estos valores parecen relativamente altos en comparación con los calculados por otros autores en Colombia y en otros países (GRECO, 2002, 57). Sin embargo, las estimaciones de este parámetro en la literatura internacional son el resultado de ejercicios econométricos aplicados no para un país sino para una muestra de países (panel data); y en el caso de Colombia, el parámetro obtenido para periodos similares a 1927-1975 dan como resultado una participación del capital en el producto muy similar a la nuestra (GRECO, 2002; Sánchez et al., 1996), y no se tiene referencia de trabajos que empleen la misma técnica de estimación (co-integración) que la aquí empleamos que permitan comparar el parámetro obtenido para el periodo 1924-2005 y el sub-periodo 1969-2005.

16 Fuente: cálculos propios. Para estimar la tasa de crecimiento del producto por trabajador se tomaron los datos de Ortiz et al (2009), GRECO (2002) y DANE. 
entre 1924-1979, se debió al progreso tecnológico y al capital; aunque fundamentalmente al progreso tecnológico. Obsérvese en la tabla 6 (columnas 1 y $4)$, que el crecimiento del producto por trabajador $(2,46 \%)$ se aproxima a la tasa de progreso tecnológico $(2,17 \%)^{17}$.

Segundo, la desaceleración observada en el crecimiento del producto por trabajador en Colombia, a partir de finales de década de 1970, conocida como de desindustrialización (Ortiz et al., 2009), se debió tanto a la desaceleración de la acumulación de capital, como de la tasa del progreso tecnológico, aunque la desaceleración de la acumulación del capital por trabajador fue muy importante. Obsérvese en la tabla 6 que hay una caída en el crecimiento de ambas variables (columnas 3 y 6); sin embargo, la disminución del producto por trabajador $(-1.48 \%)$, medida como la diferencia entre las columnas 2 y 1 , es mayor que la disminución de la tasa de progreso tecnológico $(-0.59 \%)$, medida como la diferencia entre las columnas 5 y 4 . Esto es lo que se espera cuando la disminución del crecimiento por trabajador es explicada en parte por la disminución del capital por trabajador; de hecho, la variación de la desaceleración del crecimiento del capital por trabajador entre los dos sub-periodos de análisis fue de $-1.41 \%$ (ver tabla 4 e Ilustración 2).

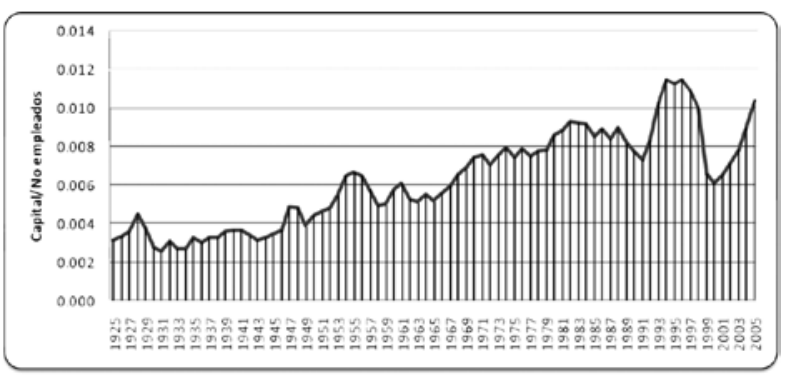

Ilustración 2. Formacion interna bruta de capital físico por trabajador (fibkf/no empleados), 1925-2005. (Millones de pesos de 1975). ${ }^{18}$

\section{Cambio técnico y crecimiento}

Una vez definida la tasa de crecimiento del progreso tecnológico y su papel en el crecimiento del producto por trabajador (productividad laboral), nos interesa ahora determinar de manera específica la participación del cambio técnico (y del capital y la PEA) en el crecimiento del producto total.

La medición del cambio técnico o PTF, su efecto en el crecimiento, y sus determinantes no es nueva en la literatura ${ }^{19}$. En Colombia, los trabajos evalúan la PTF a nivel agregado y a nivel de firmas. Algunos trabajos con un enfoque macroeconómico son los de Sánchez et al. (1996) y GRECO (2002), y algunos trabajos con un enfoque microeconómico son los de Medina, Meléndez \& Seim (2002); Fernandes (2003); Eslava, Haltiwanger, Kugler \& Kugler (2004); y Echavarría, González \& Villamizar (2004). La literatura coincide, en general, en que la PTF tiene una relación directa con el crecimiento. Sin embargo, la evidencia de sus efectos sobre el crecimiento en Colombia es contradictoria, al igual que la evidencia aportada sobre sus determinantes (González, 2004).

Continuando con el enfoque Solow-Swan, podemos suponer que el crecimiento del PIB real (Y) está determinado por el capital real agregado disponible $(K)$, una medida del trabajo ( $L)$, y un factor de cambio técnico $(A)$ que afecta al trabajo ${ }^{20}$ (véase, por ejemplo, Solow, 1956; Barro \& Sala-i-Martin, 1995; y para Colombia, Sánchez et al., 1996; Posada, 1993; y GRECO, 2002). Formalmente, en términos de una función de producción Cobb-Douglas, donde el parámetro $\alpha$ es una medida de la elasticidad del capital sobre el producto, tenemos:

$Y=K \propto(A L) 1-\propto ; 0<\alpha<1$

Para determinar empíricamente la participación en la explicación de $Y$ de cada uno de sus determinantes,

\footnotetext{
Aunque mayor que la diferencia en el periodo completo: una diferencia de $0.29 \%$, frente a $0.05 \%$ (tabla 5). Fuente: Cálculos propios con base en datos de Ortiz et al. (2009).

Un resumen de las metodologías de estimación y determinantes de la productividad en Colombia se encuentra en González (2004).

Bajo el supuesto de progreso técnico neutral en el sentido de Harrod (Romer, 1996).
} 
suponiendo que todas las variables son continuas en el tiempo, se saca logaritmos y se deriva con respecto al tiempo. Así el crecimiento del cambio técnico o crecimiento de todos los factores diferentes al capital y al trabajo que afectan al crecimiento:

$A=11-\propto Y-\propto 1-\propto K-L$

De [12] podemos estimar la tasa de crecimiento del cambio técnico. Sin embargo, las variables son tomadas en términos agregados, mientras en la parte (2) son tomadas por trabajador. Finalmente, si el cambio técnico recoge básicamente el conocimiento, estaríamos estimando el efecto del conocimiento sobre el crecimiento del producto total de la economía.

Con base en la ecuación [12] y los datos de la tabla 4 y 5 , en la tabla 7 se presenta la tasa de crecimiento del cambio técnico para todo el periodo de análisis y los dos sub-periodos que se han venido analizando.

Tabla 7. La contabilidad del cambio técnico colombiano, 1905-2005. ${ }^{21}$

\begin{tabular}{|c|c|c|c|c|}
\hline \multirow{2}{*}{$\begin{array}{c}\text { Periodos } \\
\text { 1925-1979 }\end{array}$} & $\begin{array}{c}{[1 /(1-\alpha)] \triangle \mathrm{PIB}} \\
(1)\end{array}$ & $\begin{array}{c}{[\alpha /(1-\alpha)] \triangle K} \\
(2)\end{array}$ & $\begin{array}{c}\Delta \mathrm{PEA} \\
(3)\end{array}$ & $\begin{array}{c}\Delta \mathrm{A} \\
(4)=(1)-(2+3)\end{array}$ \\
\cline { 2 - 5 } & 8,79 & 3,04 & 2,35 & 3,40 \\
\hline $1969-2005$ & 11,33 & 7,51 & 3,41 & 0,41 \\
\hline $1925-2005$ & 12,97 & 7,57 & 2,70 & 2,70 \\
\hline
\end{tabular}

Los datos muestran un crecimiento positivo del cambio técnico en Colombia (2,7\%) para todo el periodo de análisis (1925-2005). No obstante, el crecimiento del cambio técnico fue más importante a comienzos que a finales de siglo, lo que muestra una preocupante tendencia decreciente: de un crecimiento de 3,4\% entre 1925-1979 se pasó a un 0,4\% entre 1969 y 2005. En la tabla (8) se presenta la participación del cambio técnico, del capital y el trabajo, en el crecimiento del producto total colombiano, para el periodo total de análisis, 1924-2005, y los dos subperiodos analizados.
Tabla 8. Estimación del crecimiento colombiano, 19242005. (variaciones porcentuales) ${ }^{22}$

\begin{tabular}{|c|c|c|c|c|}
\hline $\begin{array}{c}\text { Periodos } \\
\text { 1924-1979 }\end{array}$ & $\begin{array}{c}\Delta \mathrm{PIB} \\
(1)=(2+3+4)\end{array}$ & $\begin{array}{c}\alpha \Delta K \\
(2)\end{array}$ & $\begin{array}{c}(1-\alpha) \triangle P E A \\
(3)\end{array}$ & $\begin{array}{c}(1-\alpha) \triangle A \\
(4)\end{array}$ \\
\cline { 2 - 5 } & 4,81 & 1,67 & 1,29 & 1,86 \\
\hline $1969-2005$ & 4,37 & 2,90 & 1,32 & 0,16 \\
\hline $1924-2005$ & 4,48 & 2,61 & 0,93 & 0,93 \\
\hline
\end{tabular}

Los datos muestran, primero, que el cambio técnico en Colombia ha jugado un papel importante en el crecimiento del producto total, pero este papel ha venido disminuyendo, pasó de un $1,86 \%$ entre 1924-1979 a un 0,16\% entre 1969-2005. Segundo, el cambio técnico fue el factor más importante en la explicación del crecimiento del producto total colombiano entre 1924-1979, con una participación de $1,86 \%$ de un crecimiento promedio total del producto de $4,8 \%$. Tercero, debido a la disminución de su tasa de crecimiento (tabla 7), el cambio técnico pasó a un segundo plano en la explicación del crecimiento del producto total para el periodo 1969-2005, explica sólo el $0,16 \%$ de un crecimiento total del producto de $4,4 \%$. Cuarto, la pérdida de la participación del cambio técnico en la explicación del crecimiento del producto total, ha sido compensada, en parte, por el capital y el trabajo. Quinto, a pesar que el cambio técnico fue el principal factor que explicó el crecimiento total hasta finales de la década de 1970, su disminución a finales del siglo XX fue tan dramática que ha significado que sea el capital el principal factor que explica el crecimiento del producto total para todo el periodo de análisis.

\section{Conclusiones}

Para aproximarnos a los efectos del conocimiento sobre el crecimiento económico colombiano, en este trabajo suponemos, partiendo de Solow y Swan, que el capital, el trabajo y el cambio técnico recogen los efectos de la actividad económica sobre el crecimiento. Siendo el cambio técnico donde se captan

21 Fuente: Elaboración propia a partir de los datos de las tablas 4 y 5 .

22 Fuente: Cálculos propios con base en los datos de las tablas 4,5 y 7. 
o recogen los efectos del conocimiento sobre el crecimiento de la economía (y de las demás variables distintas a capital y trabajo). Así las cosas, las estimaciones de la parte (2) son una buena aproximación de la dinámica y efecto del conocimiento sobre el producto por trabajador o productividad del trabajo; y las estimaciones de la parte (3) son una buena medida de la participación del conocimiento sobre el crecimiento del producto total de la economía.

Es claro, sin embargo, que la estimación de la participación del cambio técnico en el producto total, al recoger el efecto del conocimiento y de otras variables distintas al capital y al trabajo, distorsiona la verdadera participación del conocimiento sobre el producto total. De igual manera, la tasa de crecimiento del progreso tecnológico al recoger la dinámica del conocimiento y de las demás variables distintas del capital, distorsiona su efecto sobre el crecimiento del producto por trabajador. No obstante, la evidencia internacional muestra que el crecimiento reciente de las economías modernas es explicado, básicamente, por la materialización de los avances de la ciencia y la tecnología en la actividad productiva, es decir, por el conocimiento ${ }^{23}$. Así las cosas, con los modelos aquí estimados, el cambio técnico estaría recogiendo básicamente la participación del conocimiento en el crecimiento del producto total, y la tasa del progreso tecnológico estaría midiendo, básicamente, la dinámica del conocimiento y su efecto sobre la productividad del trabajo o producto por trabajador.

Bajo estas consideraciones y de acuerdo con la información y el análisis realizado, se pueden sacar las siguientes conclusiones generales:

- En Colombia históricamente se ha aprovechado el conocimiento a través de la materialización de la ciencia y la tecnología en la actividad productiva; sin embargo, la tendencia es que se aprovecha cada vez menos este factor. Esta situación ha llevado a que el conocimiento haya tenido una participación importante en el cre- cimiento y comportamiento del producto total y por trabajador, y explica también por qué dicha participación ha venido disminuyendo en el tiempo.

- El uso o materialización del conocimiento en la actividad productiva, y la tendencia decreciente de esta situación, explica, en parte, el despegue y crecimiento observado del producto total y por trabajador de la economía desde comienzos del siglo XX y hasta finales de la década de 1970, así como parte de la disminución del crecimiento observada por un poco más de las tres últimas décadas de este mismo siglo.

- El crecimiento del producto por trabajador observada desde 1924 y hasta finales de la década de 1970 en Colombia, es explicado tanto por la dinámica de la materialización del conocimiento en la actividad productiva como por la mayor inversión de capital por trabajador; pero fue la dinámica del uso del conocimiento en la actividad productiva el factor que jugó el papel principal.

- La desaceleración del producto por trabajador observada desde finales de la década de 1970 y hasta finales del siglo XX, es explicada tanto por la desaceleración del capital por trabajador ${ }^{24}$ (tabla 4 y Ilustración 1) como por la desaceleración de la materialización del conocimiento en la actividad productiva. Este es un indicativo de la dependencia cada vez mayor del crecimiento colombiano del capital.

- La disminución de la dinámica de la materialización del conocimiento en la actividad productiva ha significado que el crecimiento colombiano dependa cada más del uso intensivo del capital y no tanto de la aplicación de los conocimientos en la actividad productiva, que aumente la productividad del trabajo. En este marco, la leve

23 Solow, 1957; Dosi et al, 1993; OECD, 1996; Vilaseca \& Torrent, 2005; Torrent, 2009; Barceló, 2001; CEPAL, 2008, 2009; CEPAL y Naciones Unidas, 2009; Banco Mundial, 2003; Corona, 2010; López \& Corona, 2010, entre otros.

24 Esto puede estar asociado con el incremento de la violencia: surgimiento de los grupos guerrilleros, el narcotráfico, secuestros, etc. (Cárdenas, 2002; Ortiz, 2009). Pero básicamente con los secuestros, ya que estos afectan principalmente a las personas de más altos ingresos (Ortiz, 2009). 
recuperación del crecimiento colombiano observada en los primero años del siglo XXI, es explicada básicamente por el aumento de la inversión del capital por trabajador en este mismo periodo (Ilustración 1) y no tanto por la recuperación de la dinámica del uso del conocimiento en la actividad productiva.

- La disminución de la participación del conocimiento en la explicación del crecimiento en Colombia, contrasta con lo observado desde las dos últimas décadas del siglo pasado en las economías más desarrolladas y que presentan las mayores tasas de crecimiento. Mientras el conocimiento tecnológico ha venido ganando paulatinamente importancia en la explicación del crecimiento de las economías con mayores tasas de crecimiento en el mundo hasta convertirse en el factor clave de su crecimiento, en Colombia este factor ha venido perdiendo paulatinamente importancia como factor clave del crecimiento.

- Los resultados aportan evidencia en favor de la hipótesis de que en Colombia, el producto por trabajador en el largo plazo depende de factores distintos al capital, en particular de la dinámica del conocimiento. En otras palabras, en el largo plazo los efectos del capital sobre el crecimiento del producto por trabajador se agotan y este último crece a la tasa que crece el progreso tecnológico; es decir, es la dinámica de la innovación la que explica la productividad del trabajo en el largo plazo en Colombia.

- Desde la política económica del desarrollo y el bienestar, esta situación es preocupante debido a que si quiere entrar en una senda de crecimiento alta y sostenida en el largo plazo, debe ser la dinámica de la materialización del conocimiento en la actividad productiva la que sustente el crecimiento.

\section{Referencias}

Aghion, P. \& Howitt, P. (1998). Endogenous growth theory. The MIT Press. Cambridge, Massachusetts, London England.

Aghion, P. \& Howitt, P. (2006). Appropriate growth policy: a unifying framework. En: Journal of the European Economic Association April-May 2006 4(2-3):269-314.
Amable B. (2003). The Diversity of Modern Capitalism. Oxford University Press.

Arrow, K. J. (1962). The Economic Implication of Learning by Doing. En: Review of Economic Studies. 29, 155-173.

Banco Mundial (2003). Aprendizaje permanente en la economía global del conocimiento. Desafíos para los países en desarrollo. Alfaomega.

Barceló, M. (2001). Hacia una economía del conocimiento. Escuela superior de gestión comercial y marketing ESIC, Price water house coopers.

Barro, R. \& Sala-i-martin, X. (1995). Economic Growth. McGraw-Hill international editions.

Blanchard, O. (2008). Macroeconomía. Pearson - Prentice Hall cuarta edición.

Benavides, O. (2010). La innovación tecnológica desde una perspectiva evolutiva: teoría e historia económica. En: Innovación. Desafío para el desarrollo en el siglo XXI. Universidad Nacional de Colombia.

Cárdenas. M. (2002). Economic Growth in Colombia: A Reversal of 'Fortune'? En: Archivos de Economía 178.

CEPAL. (1999). La CEPAL y las nuevas teorías del crecimiento. Revista de la CEPAL 68.

CEPAL (2008a). Espacios Iberoamericanos. La economía del conocimiento. En: Revista de la CEPAL.

CEPAL (2008b). La economía del conocimiento. Indicadores varios sobre economía del conocimiento. En: Revista de la CEPAL.

CEPAL. (2009). Innovar para crecer. Desafíos y oportunidades para el desarrollo sostenible e inclusivo en Iberoamérica. Disponible en: www.eclac.org/

CEPAL \& Naciones Unidas (2009). Innovar para crecer. Desafíos y oportunidades para el desarrollo sostenible e inclusivo en Iberoamérica.

Corona. L. (2010). Innovación ante la sociedad del conocimiento. Disciplinas y enfoques. Plaza y Valdés editores. UNAM, Facultad de Economía

Dosi, G., Pavitt, K., \& Soete, L. (1993). La economía del cambio técnico y el comercio internacional. Conacyt, SECOFI.

Echavarría, J., González J. \& Villamizar, M. (2004). El Crecimiento Industrial y sus Determinantes 1975-2002. Mimeo.

Eslava. M., Haltiwanger J., Kugler A. \& Kugler M. (2004). "The effect of structural reforms on productivity and profitability enhancing reallocation: Evidence from Colombia" En: NBER Working Paper No.10367

Fernandes, A. (2003). Trade Policy, Trade Volumes and Plant-Level Productivity in Colombian Manufacturing Industries. Universidad de Yale. Mimeo.

González, J. (2004). Productividad: metodologías de estimación y determinantes en Colombia. Disponible en: Webpondo.org.

GRECO (2002). El crecimiento económico colombiano en el siglo XX. Banco de la república. Fondo de Cultura Económica.

Grossman, G. M \& Helpman, E. (1991). Innovation and Growth in the Global Economy. Cambridge Massachusetts, the MIT Press.

Johansen.S. (1988). Statistical analysis of cointegration vectors. En: Journal of economic dynamics and control, 12, 231-254.

Johansen. S. (1995). Likelihood-based inference in cointegrated vector autoregressive models. Oxford University Press.

López. S., \& Corona. T. (coord) (2010). Gestión y Políticas del conocimiento y la innovación. Universidad Autónoma de Sinaloa. México.

Lucas, R. (1998). On the mechanics of economic development. En: Journal of Monetary Economics.Vol.22, págs.3-42. 
Marx C. (1984). El capital. Vol. I. Fondo de Cultura Económica. Decimoctava reimpresión.

Medina, P., Melendez M. \& Seim K. (2002) "Productivity Dynamics of the Colombian Manufacturing Sector" En: Documento CEDE, Universidad de los Andes.

Morín, E. (1988). El método, tomo III. Cátedra, Barcelona.

OECD (1996). The Knowledge Based Economy. Disponible en: www.oecd.org/

Observatorio Colombiano de Ciencia Tecnología -OCCyT- (2004). Indicadores de Ciencia y Tecnología, Colombia. Capítulo 9.

Observatorio Colombiano de Ciencia y Tecnología-OCCyT- (2009). Indicadores de Ciencia y Tecnología. Colombia.

Obstfeld M., \& Rogoff K. (1996). Foundations of international macroeconomics. MIT Press, Cambridge (Ma).

Ocampo, J. (1999) (Compilador). Historia Económica de Colombia. T.M Editores. FEDESARROLLO. Cuarta Edición.

Ortiz, C. Uribe, Vivas, J. (2009). Transformación industrial, autonomía tecnológica y crecimiento económico. Colombia 1925 - 2005. En: Archivos de Economía, documento 352. DNP.

Ortiz, C. (2009). La desaceleración económica colombiana: se cosecha lo que se siembra. En: Revista de Economía Institucional, 11, (21): 107-137.

Posada. C. (1993). Productividad, crecimiento y ciclos en la economía colombiana (1967-1992). En: Archivos de Macroeconomía (DNP), No. 16.

Ray, D. [1998]. Economía del desarrollo. Antoni Bosch editor.

Romer, P. (1986). Increasing Returns and Long-Run Growth. En: Journal of Political Economy, 94 (5): 1002-1037.

Romer, P. (1990). Endogenous Technical Change. Journal of Political Economy. 98 (5): 71-102.

Salazar, M. (2010). Una aproximación a la innovación desde la evolución de la política de ciencia y tecnología. En: Innova- ción. Desafío para el desarrollo en el siglo XXI. Universidad Nacional de Colombia.

Sánchez, F. Rodríguez, J. \& Núñez, J. (1996). Evolución y determinantes de la productividad en Colombia: Un análisis global y sectorial. En: Archivos de Macroeconomía, documento 50. D.N.P.

Schumpeter, J. (1934). The Theory of Economic Development. Nueva York: Oxford University Press.

Schultz. T. W. (1961). Investment in human capital. En: American Economic Review: 1 - 17.

Sen, A. (1987). Commodities and capabilities. Oxford: Oxford University Press.

Sen, A. (1996). Capacidad y bienestar. En Nussbaum, M. \& Sen, A. (Comp.).La calidad de vida. (Traducción de Roberto Reyes Mazzoni). México: Fondo de Cultura Económica. (Trabajo original publicado en 1993).

Sen, A. (2000). Desarrollo y libertad. Bogotá: Planeta.

Smith, A. (1992). La riqueza de las naciones. Fondo de Cultura Económica, séptima reimpresión.

Solow, R. (1956). A contribution to the theory of economic Growth. En: Quarterly journal economics, No.70, I (febrero).

Solow, R. (1957). Technical change and the aggregate production function. En: The Review of Economics and Statistics, 39 (3): $312-320$

Swan, T. (1956). Economic growth and capital accumulation. Economic record, 32.

Torrent, J. (2009). Conocimiento, redes y actividad económica: un análisis de los efectos de red en la economía del conocimiento. En: UOC, revista sobre la sociedad del conocimiento. Disponible en: http://uocpapers.uoc.edu.

Vilaseca. J. \& Torrent, J. (2005). TIC, conocimiento y crecimiento económico. Un análisis empírico, agregado e internacional, sobre las fuentes de la productividad. Disponible en: www. mityc.es/Publicaciones/.../3P41-60_\%20Ei\%20360-7.pdf 


\section{Anexos 1}

Resultados de la prueba de raíz unitaria del logaritmo natural del producto por trabajador: LNYPEA

Null Hypothesis: LNYPEA has a unit root

Exogenous: Constant

Lag Length: 1 (Automatic based on SIC, MAXLAG=11)

\begin{tabular}{lccc}
\hline & t-Statistic & Prob. $^{*}$ \\
\hline Augmented Dickey-Fuller test statistic & -2.030112 & 0.2736 \\
\hline Test critical values: & $1 \%$ level & -3.514426 & \\
\hline & $5 \%$ level & -2.898145 & \\
$10 \%$ level & -2.586351 &
\end{tabular}

*MacKinnon (1996) one-sided p-values.

Augmented Dickey-Fuller Test Equation

Dependent Variable: D(LNYPEA)

Method: Least Squares

Date: 09/02/11 Time: 15:24

Sample (adjusted): 19262005

Included observations: 80 after adjustments

\begin{tabular}{lcccl}
\hline & Coefficient & Std. Error & t-Statistic & Prob. \\
\hline LNYPEA(-1) & -0.015018 & 0.007398 & -2.030112 & 0.0458 \\
D(LNYPEA(-1)) & 0.300645 & 0.106754 & 2.816244 & 0.0062 \\
C & -0.037151 & 0.023928 & -1.552612 & 0.1246 \\
\hline R-squared & 0.168278 & Mean dependent var & 0.016814 \\
Adjusted R-squared & 0.146675 & S.D. dependent var & 0.030557 \\
S.E. of regression & 0.028228 & Akaike info criterion & -4.260252 \\
Sum squared resid & 0.061354 & Schwarz criterion & -4.170926 \\
Log likelihood & 173.4101 & Hannan-Quinn criter. & -4.224438 \\
F-statistic & 7.789492 & Durbin-Watson stat & 1.952847 \\
Prob(F-statistic) & 0.000830 & & \\
\hline
\end{tabular}

\section{Resultados de la prueba de raíz unitaria del logaritmo natural del capital por trabajador: LNKPEA}

Null Hypothesis: LNKPEA has a unit root

Exogenous: Constant

Lag Length: 1 (Automatic based on SIC, MAXLAG=11)

\begin{tabular}{lccc}
\hline & t-Statistic & Prob. $^{*}$ \\
\hline Augmented Dickey-Fuller test statistic & -1.596359 & 0.4797 \\
\hline Test critical values: & 1\% level & -3.515536 & \\
& 5\% level & -2.898623 & \\
& $10 \%$ level & -2.586605 & \\
\hline
\end{tabular}

*MacKinnon (1996) one-sided p-values.

Augmented Dickey-Fuller Test Equation

Dependent Variable: D(LNKPEA)

Method: Least Squares

Date: 03/26/11 Time: 10:09

Sample (adjusted): 19272005

Included observations: 79 after adjustments

\begin{tabular}{lcccl}
\hline & Coefficient & Std. Error & t-Statistic & Prob. \\
\hline LNKPEA(-1) & -0.046218 & 0.028952 & -1.596359 & 0.1146 \\
D(LNKPEA(-1)) & 0.276613 & 0.110313 & 2.507522 & 0.0143 \\
C & -0.229357 & 0.150456 & -1.524406 & 0.1316 \\
\hline R-squared & 0.096977 & Mean dependent var & 0.013073 \\
Adjusted R-squared & 0.073213 & S.D. dependent var & 0.117898 \\
S.E. of regression & 0.113500 & Akaike info criterion & -1.476795 \\
Sum squared resid & 0.979049 & Schwarz criterion & -1.386816 \\
Log likelihood & 61.33340 & Hannan-Quinn criter. & -1.440746 \\
F-statistic & 4.080871 & Durbin-Watson stat & 1.877859 \\
Prob(F-statistic) & 0.020728 & & \\
\hline
\end{tabular}




\section{Anexo 2:}

\section{DESCRIPCIÓN Y FUENTES DE LA INFORMACIÓN ESTADÍSTICA}

\begin{tabular}{|c|c|c|c|c|c|c|c|}
\hline AÑO & PIB & PIBPC & PEA & ESCOL & FIBKF & РОВ & EMPLEO \\
\hline 1924 & 8.637 & 1.255 & 2.451 .651 & 32,8 & & 6882 & 2327068 \\
\hline 1925 & 8.860 & 1.255 & 2.501 .202 & 33,2 & 7441,5 & 7061 & 2370510 \\
\hline 1926 & 9.707 & 1.340 & 2.547 .447 & 33,6 & 8100,7 & 7243 & 24140140 \\
\hline 1927 & 10.581 & 1.424 & 2.593 .008 & 33,9 & 8790,9 & 7431 & 2456624 \\
\hline 1928 & 11.357 & 1.490 & 2.642 .496 & 34 & 11240,4 & 7624 & 2502994 \\
\hline 1929 & 11.768 & 1.505 & 2.690 .895 & 34,6 & 9653,7 & 7821 & 2548416 \\
\hline 1930 & 11.666 & 1.474 & 2.741 .281 & 35,2 & 7173 & 7914 & 2595732 \\
\hline 1931 & 11.595 & 1.448 & 2.797 .594 & 35,5 & 6768,5 & 8009 & 2648726 \\
\hline 1932 & 12.243 & 1.511 & 2.855 .992 & 36,1 & 8341,1 & 8104 & 2703612 \\
\hline 1933 & 12.930 & 1.577 & 2.915 .765 & 37 & 7444,7 & 8201 & 2759444 \\
\hline 1934 & 12.661 & 1.526 & 2.976 .224 & 31 & 7478,8 & 8299 & 2816223 \\
\hline 1935 & 14.080 & 1.677 & 3.038 .758 & 29 & 9283,8 & 8398 & 2874894 \\
\hline 1936 & 14.824 & 1.744 & 3.103 .442 & 32 & 8877,3 & 8498 & 2935458 \\
\hline 1937 & 15.055 & 1.751 & 3.166 .849 & 33 & 9770,4 & 8599 & 2995975 \\
\hline 1938 & 16.038 & 1.843 & 3.234 .430 & 34 & 10018,3 & 8702 & 3058478 \\
\hline 1939 & 17.020 & 1.905 & 3.296 .286 & 35 & 11042,8 & 8935 & 3110525 \\
\hline 1940 & 17.386 & 1.895 & 3.359 .598 & 35 & 11545,4 & 9174 & 3163519 \\
\hline 1941 & 17.681 & 1.877 & 3.425 .242 & 35 & 11805,2 & 9419 & 3218405 \\
\hline 1942 & 17.713 & 1.832 & 3.491 .994 & 36 & 11017,4 & 9671 & 3274237 \\
\hline 1943 & 17.790 & 1.792 & 3.559 .868 & 40 & 10477 & 9930 & 3331016 \\
\hline 1944 & 18.991 & 1.863 & 3.631 .269 & 40 & 11079,7 & 10196 & 3390634 \\
\hline 1945 & 19.883 & 1.899 & 3.703 .933 & 36 & 11824,1 & 10469 & 3451198 \\
\hline 1946 & 21.681 & 2.017 & 3.762 .755 & 46 & 12854,6 & 10749 & 3498513 \\
\hline 1947 & 22.535 & 2.042 & 3.824 .904 & 47 & 17213,6 & 11036 & 3548668 \\
\hline 1948 & 23.235 & 2.050 & 3.889 .375 & 42 & 17369,9 & 11332 & 3600715 \\
\hline 1949 & 24.519 & 2.107 & 3.952 .926 & 48 & 14186 & 11635 & 3651816 \\
\hline 1950 & 24.955 & 2.153 & 4.019 .784 & 56 & 16408,6 & 11591,658 & 3705755 \\
\hline 1951 & 25.726 & 2.150 & 4.076 .863 & 64 & 17489,1 & 11965 & 3821161 \\
\hline 1952 & 27.350 & 2.214 & 4.158.392 & 65 & 19182,1 & 12350,771 & 4013543 \\
\hline 1953 & 29.026 & 2.277 & 4.241 .550 & 66 & 22326,5 & 12749,619 & 4105470 \\
\hline 1954 & 31.042 & 2.358 & 4.326 .372 & 70 & 27428,8 & 13162,003 & 4251740 \\
\hline 1955 & 32.242 & 2.373 & 4.408.342 & 77 & 29039,7 & 13588,405 & 4342497 \\
\hline 1956 & 33.539 & 2.391 & 4.503 .656 & 93 & 29045,5 & 14029,321 & 4485172 \\
\hline 1957 & 34.766 & 2.400 & 4.601.032 & 108 & 26307,8 & 14485,993 & 4567291 \\
\hline 1958 & 35.639 & 2.383 & 4.700 .512 & 115 & 22780,8 & 14958,277 & 4634734 \\
\hline 1959 & 38.207 & 2.473 & 4.802.144 & 128 & 24022,2 & 15446,73 & 4834734 \\
\hline 1960 & 39.831 & 2.497 & 4.902.415 & 140 & 28147,3 & 15952,727 & 4907838 \\
\hline 1961 & 41.847 & 2.540 & 5.030 .317 & 156 & 31107 & 16476,124 & 5110894 \\
\hline
\end{tabular}




\begin{tabular}{|c|c|c|c|c|c|c|c|}
\hline 1962 & 44.120 & 2.594 & 5.161 .556 & 175 & 28183,1 & 17009,885 & 5352708 \\
\hline 1963 & 45.571 & 2.597 & 5.296 .219 & 202 & 27852,3 & 17546,017 & 5459094 \\
\hline 1964 & 48.389 & 2.675 & 5.434 .696 & 229 & 31580,2 & 18090 & 5759094 \\
\hline 1965 & 50.136 & 2.689 & 5.568 .284 & 266 & 30766 & 18646,175 & 5968428 \\
\hline 1966 & 52.806 & 2.750 & 5.740 .469 & 320 & 35025 & 19201,732 & 6261077 \\
\hline 1967 & 55.028 & 2.784 & 5.917 .978 & 377 & 38102 & 19764,027 & 6371398 \\
\hline 1968 & 58.398 & 2.874 & 6.100 .976 & 447 & 43353 & 20321,665 & 6633743 \\
\hline 1969 & 62.116 & 2.976 & 6.289 .633 & 523 & 47457 & 20869,14 & 6893712 \\
\hline 1970 & 66.308 & 3.094 & 6.483 .890 & 750,1 & 53201 & 21429,658 & 7138886 \\
\hline 1971 & 70.250 & 3.194 & 6.750 .268 & 846,2 & 55786 & 21992,579 & 7378696 \\
\hline 1972 & 75.637 & 3.355 & 7.027 .590 & 954,6 & 54687 & 22542,89 & 7748163 \\
\hline 1973 & 80.728 & 3.499 & 7.316 .306 & 1076,9 & 59443 & 23069,231 & 78641172 \\
\hline 1974 & 85.370 & 3.618 & 7.577 .147 & 1214,9 & 64604 & 23593,39 & 8117425 \\
\hline 1975 & 87.347 & 3.622 & 7.836 .895 & 1370,6 & 62129 & 24114,177 & 8377876 \\
\hline 1976 & 91.488 & 3.716 & 8.106 .476 & 1436,4 & 68039 & 24620,486 & 8628428 \\
\hline 1977 & 95.283 & 3.797 & 8.385 .330 & 1505,5 & 68518 & 25094,412 & 9112372 \\
\hline 1978 & 103.366 & 4.047 & 8.673 .777 & 1577,9 & 74923 & 25542,727 & 9655789 \\
\hline 1979 & 108.906 & 4.184 & 8.972 .146 & 1653,7 & 77775 & 26030,848 & 9967570 \\
\hline 1980 & 113.375 & 4.265 & 9.270 .861 & 1733,2 & 88021 & 26582,811 & 10241749 \\
\hline 1981 & 115.789 & 4.263 & 9.569 .363 & 1768,1 & 93539 & 27159,381 & 10601876 \\
\hline 1982 & 116.938 & 4.212 & 9.877 .476 & 1816,6 & 96307 & 27764,644 & 10395038 \\
\hline 1983 & 118.806 & 4.185 & 10.195 .509 & 1889,6 & 97444 & 28389,398 & 10564662 \\
\hline 1984 & 123.037 & 4.239 & 10.523 .782 & 1889 & 98656 & 29027,923 & 10764662 \\
\hline 1985 & 127.076 & 4.282 & 10.855 .203 & 1934 & 93505 & 29678,395 & 10949767 \\
\hline 1986 & 134.844 & 4.446 & 11.238 .180 & 2136,2 & 100650 & 30326,818 & 11292166 \\
\hline 1987 & 142.086 & 4.589 & 11.634 .670 & 2187,8 & 101471 & 30964,245 & 12084825 \\
\hline 1988 & 147.896 & 4.682 & 12.045 .148 & 2235,3 & 112502 & 31589,376 & 12523926 \\
\hline 1989 & 152.686 & 4.739 & 12.470 .108 & 2282,8 & 106611 & 32216,557 & 13044064 \\
\hline 1990 & 159.042 & 4.840 & 12.887 .441 & 2330,4 & 103046 & 32858,579 & 13274935 \\
\hline 1991 & 161.587 & 4.821 & 13.322 .910 & 2377,9 & 102745 & 33518,61 & 14042304 \\
\hline 1992 & 167.889 & 4.909 & 13.773 .093 & 2686,5 & 117276,4 & 34202,721 & 14127667 \\
\hline 1993 & 175.444 & 5.028 & 14.238 .488 & 2796 & 151337 & 34896,573 & 14674507 \\
\hline 1994 & 186.496 & 5.240 & 14.444 .920 & 2935,8 & 169672,1 & 35588,746 & 14837769 \\
\hline 1995 & 196.567 & 5.418 & 14.912 .046 & 3025,4 & 171208,5 & 36280,883 & 15169690 \\
\hline 1996 & 200.695 & 5.428 & 15.231 .034 & 3317,8 & 168818,2 & 36971,016 & 14725167 \\
\hline 1997 & 203.706 & 5.409 & 15.556 .846 & 3434,3 & 165424 & 37657,83 & 15173856 \\
\hline 1998 & 205.132 & 5.350 & 12.717 .558 & 3753,7 & 155000,8 & 38339,937 & 15387990 \\
\hline 1999 & 196.722 & 5.042 & 16.979 .000 & 3801 & 101438,2 & 39016,113 & 15318712 \\
\hline 2000 & 202.230 & 5.096 & 19.413 .000 & 3781,1 & 99390,3 & 39685,655 & 16321087 \\
\hline 2001 & 205.204 & 5.086 & 19.705 .000 & 3580,2 & 107830,9 & 40349,388 & 16506004 \\
\hline 2002 & 209.163 & 5.101 & 20.093 .988 & 3936,3 & 118449,3 & 41008,227 & 16620212 \\
\hline 2003 & 217.791 & 5.228 & 20.814 .085 & 4272,5 & 136704,5 & 41662,073 & 17466865 \\
\hline 2004 & 226.183 & 5.336 & 20.479 .646 & 4384,7 & 157239,5 & 42310,775 & 17577876 \\
\hline 2005 & 234.360 & 5.434 & 20.938.997 & 4556,8 & 187025,4 & 42954,279 & 18071950 \\
\hline
\end{tabular}

